http://dx.doi.org/10.18232/alhe.1062

Artículos

\title{
Modelos de regulación de servicios públicos de gas natural en Argentina, 1967-2017
}

\section{Models of Regulation of Natural Gas Public Services in Argentina, 1967-2017}

Esteban Serrani ${ }^{1,} *$ (D) 0000-0003-2682-7738

${ }^{1}$ Universidad Nacional de San Martín, Buenos Aires, Argentina.

* Correspondencia: estebanserrani@yahoo.com.ar

Resumen. En Argentina, la privatización de las empresas de servicios públicos significó el pasaje de un modelo de regulación, basado en un monopolio estatal, a otro modelo de eficiencia económica marginalista que, sin embargo, tuvo un derrotero cambiante y contradictorio en las últimas décadas. El objetivo de este artículo es analizar las marchas y contramarchas de los diferentes modelos regulatorios del servicio público de distribución residencial de gas natural en Argentina entre 1967 y 2017. A partir de la revisión de la legislación regulatoria de cinco décadas, de la reconstrucción de series de precios de tarifas residenciales, y de la evolución de las empresas del sector en la cúpula empresarial, se analizan los efectos que tuvieron los diseños institucionales y los mecanismos para la determinación de los precios energéticos en la dinámica tarifaria, el poder adquisitivo del salario y las empresas del sector.

Palabras clave: gas natural; servicios públicos; marco regulatorio.

Abstract. In Argentina, the privatization of public service companies meant the passage from a model of regulation based on a state monopoly to another model of marginal economic efficiency, which, however, had a changing and contradictory course in the last five decades. The article aim is to analyze the marches and countermarched of the different regulatory models of the public service of residential natural gas

CÓMO CITAR: Serrani, E. (2020). Modelos de regulación de servicios públicos de gas natural en Argentina, 1967-2017. América Latina en la Historia Económica, 27(2), e1062. DOI: 10.18232/alhe.1062 
distribution in Argentina between 1967 and 2017. From the revision of the regulatory legislation of five decades, the reconstruction of prices series of residential tariffs, and the evolution of the companies of the sector in the Argentine top business, it is examined the effect of the institutional models and the determination of energy prices mechanisms on the tariff dynamics, the purchasing power of wages, and the sales of the companies in the sector.

Key words: natural gas; public services; regulatory framework.

JEL: N16; N36; N56; N76.

Recibido: 12 de febrero de 2019

Aceptado: 17 de mayo de 2019

Publicado: 17 de enero de 2020.

Organismo patrocinador: Consejo Nacional de Investigaciones Científicas y Técnicas.

\section{INTRODUCCIÓN}

La regulación de los servicios públicos es un nutrido campo académico con trabajos que ponen en diálogo abordajes disciplinarios disímiles como la economía, las ciencias políticas, la historia de las empresas públicas, y los estudios sociológicos de la administración pública. En Argentina, con las reformas estructurales de la década de 1990, no sólo se buscó la estabilización macroeconomía y la apertura comercial (Bour, 1993), sino también debilitar la participación estatal en la economía, iniciando la privatización de empresas públicas y promoviendo la desregulación de sectores del aparato productivo hasta entonces protegidos (Basualdo, 2006; Serrani, 2013). En este contexto general, los servicios públicos energéticos experimentaron una extensa transformación, como la remoción del monopolio de Estado, la fragmentación de las empresas energéticas estatales (Barrera, 2014), y la sanción de nueva normativa que modificó los marcos regulatorios vigentes (Fundación de Investigaciones Económicas Latinoamericanas, 1998; Facultad Latinoamericana de Ciencias Sociales, 1998).

El objetivo de este artículo es analizar las marchas y contramarchas de los diferentes modelos regulatorios del servicio público de distribución residencial de gas natural en Argentina entre 1967 y 2017. Se examinan la estructura y los mecanismos para la determinación de los precios energéticos para entender qué tipo de efectos tuvieron sobre la dinámica tarifaria, el poder adquisitivo del salario, y las empresas del sector. El marco metodológico utilizado es la investigación documental a través de la recolección, sistematización y análisis de diversas fuentes secundarias, como memorias y balances de la empresa Gas del Estado Sociedad del Estado (Gas del Estado), resoluciones, decretos y leyes nacionales que organizaron el marco regulatorio del sector, resoluciones del Ente Nacional Regulador del Gas (EnARGas) con los cuadros tarifarios del servicio regulado de gas natural, distintos documentos oficiales sobre el proceso de privatizaciones de las empresas estatales de servicios públicos, rankings anuales por facturación de la cúpula empresarial argentina elaborado por revistas especializadas como Mercado y Prensa Económica, actas transitorias y actas acuerdo ${ }^{1}$ firmadas entre el Estado y las distribuidoras durante el proceso de renegociación contractual instaurado en la década de 2000.

\footnotetext{
${ }^{1}$ Disponibles en Unidad de Renegociación y Análisis de Contratos de Servicios Públicos. Recuperado de http://www.uniren.gov.ar/energia.htm
} 
En el primer apartado se presenta la discusión académica alrededor de los alcances de los diferentes modelos regulatorios de monopolios naturales, al distinguir entre aquellos que aspiran a favorecer la eficiencia económica marginalista de los que buscan privilegiar la equidad distributiva del sistema. En el segundo, se revisa el modelo regulatorio de monopolio estatal ejercido por Gas del Estado, como preludio a la privatización del sistema (1967-1991). En el tercer apartado se analizan las transformaciones regulatorias desde un modelo monopólico estatal hacia uno de eficiencia marginalista (1992-2001). En el cuarto, se examinan las transformaciones del modelo de equidad distributiva, iniciado con la ley de emergencia pública tras el colapso de la convertibilidad (2002-2015). En el quinto apartado se revisa el retorno a un modelo de eficiencia marginalista a partir de la política tarifaria de eliminación gradual de subsidios de la Alianza Cambiemos (2016-2017). En el sexto, se presenta un análisis comparado de los modelos regulatorios y cómo han impactado diferencialmente en el posicionamiento de las empresas distribuidoras dentro de la cúpula empresarial argentina. Finalmente, se presentan las conclusiones del trabajo.

\section{DISGUSIONES SOBRE REGULACIÓN TARIFARIA EN MONOPOLIOS NATURALES}

El temprano desarrollo del tendido de redes de gas natural en varios países de Europa continental y la transición que significó el pasaje de fuentes fósiles altamente contaminantes y menos eficientes como el carbón y los derivados líquidos del petróleo para uso doméstico, ha sido una temática de especial atractivo para una parte considerable de la historiografía moderna.

En este marco general, destacan los estudios sobre los inicios de la industria a finales del siglo XIX en diferentes países de Europa continental (Fernández-Paradas, 2018; Sudrià, 1983), el análisis de la problemática transición de energética en la primera mitad del siglo xx (Cayón, 2001; Martínez y Mirás, 2018; Moyano, 2012), los trabajos sobre la expansión de las redes de gas, las transformaciones urbanas que trajo aparejada (Arroyo, 2015) y los cambios tecnológicos asociados en la industria gasífera (Sudrià, 1990). Por otro lado, también se han desarrollado líneas de trabajo sobre la segunda etapa de expansión de la industria del gas natural en la última mitad del siglo $\mathrm{xx}$, junto al crecimiento y difusión de las empresas nacionales de servicios públicos tanto en España (Sudrià, 1990) como en Francia (Fernández, 2018), Portugal (Matos, 2018) e Italia (Giuntini, 2009), por sólo citar algunos casos. Finalmente, es conocida la vasta tradición de estudios subnacionales del desarrollo gasífero, por ejemplo los trabajos sobre la ciudad de Barcelona (Arroyo y Matos, 2009; Moyano, 2012) y Burdeos (Fernández, 2017), o sobre regiones como Galicia (Carmona, 1999; Carmona y Pena, 1985) o Andalucía (Fernández-Paradas, 2005).

Si bien gran parte de estos estudios ha puesto el foco de análisis en diversos aspectos del sector, en su generalidad no ha reparado en las transformaciones regulatorias y tarifarias de largo plazo como objeto de análisis. Para abordar la especificidad de los objetivos planteados en este artículo, resulta necesario enfatizar en aportes multidisciplinarios que han trabajado sobre la regulación de mercados que prestan servicios públicos. En este sentido, los modelos de organización institucional diseñados para la prestación de servicios públicos estuvieron mayoritariamente orientados a estudiar cómo se regulan monopolios naturales (Beesley y Littlechild, 1989; Laffont y Tirole, 1986; Posner, 1999), es decir, mercados operados por un único oferente y cuya producción puede depender de un solo recurso que requiere de una inversión que es prohibitivamente alta para ser duplicada por una o más empresas (Bös, 1994). Las líneas de suministro de agua, los sistemas ferroviarios, las líneas de transmisión de electricidad, la estructura de gasoductos troncales, o la red urbana de distribución de gas natural, son ejemplos de monopolios naturales. 
Sin embargo, no siempre los mercados de servicios públicos se configuraron alrededor de monopolios naturales. No son pocos los autores que asumen que es posible establecer mercados de competencia (formalmente perfecta) en la prestación de estos servicios, aunque no estén exentos de problemas de competencia y eficiencia (Bustos y Galetovic, 2002; Estache, Guasch y Trujillo, 2003; Fundación de Investigaciones Económicas Latinoamericanas, 1998; Laffont y Tirole, 1993; Littlechild, 1983, 2003; Waddams y Zhu, 2013). La mayor parte de este grupo de autores identifica la intervención empresaria del Estado como vector central que promueve fallas de mercado ante la imposibilidad de poder satisfacer, simultáneamente, beneficios medios de largo plazo, una estructura óptima de precios y servicios fundada en la Ley de Pareto (precios iguales a los costos marginales bajo una operación eficiente), y que la estructura de precios no sea afectada directa o indirectamente por la conducta del regulador, implicando que las empresas la modifiquen con base en decisiones propias (Fundación de Investigaciones Económicas Latinoamericanas, 1998, p. 52).

Más allá de las diferentes teorías y escuelas alrededor de los modelos institucionales, este artículo se centrará en la determinación y evolución de la regulación tarifaria de servicios públicos. En efecto, las discusiones alrededor de cuál debe ser el mecanismo y los fundamentos para la fijación de la tarifa de un servicio público regularmente está vinculada con la necesidad de dirimir la tensión existente entre dos aspectos que son, en algún sentido, contradictorios: la equidad distributiva y la eficiencia económica (marginalista) del sistema.

En primer lugar, y en términos normativos, los modelos de regulación tarifaria que se centran fundamentalmente en la búsqueda de la eficiencia económica tienden a generar sistemas donde las empresas generadoras del bien público son remuneradas bajo un modelo de costo marginal. Es decir, la tarifa final es fijada por el costo de la última unidad que entra en funcionamiento, asumiendo que primero se van conectando al despacho las empresas que generan en forma más barata y, luego, a medida que la demanda lo requiere, entran al sistema las menos eficientes económicamente (es decir las que producen el mismo bien a un costo más elevado). En este modelo, volverse más eficiente es producir más barato, y resultaría en que, en el nivel microeconómico, las empresas busquen bajar costos para tener prioridad en el despacho, y que, en el nivel sistemático, el precio final del servicio tienda a reflejar el resultado de la competencia por bajar los costos del sistema en una carrera a la baja entre las empresas, al beneficiarse los consumidores por una caída en la tarifa final. Bajo este modelo de búsqueda de eficiencia económica a través de tarifas que reflejen el costo marginal, subyace el fundamento de que, al asegurar la rentabilidad individual y de largo plazo de las empresas, el sistema asegurará la competencia de mercado y la eficiente asignación de recursos, con el fin de que la tarifa exprese el precio de equilibrio del mercado.

En segundo lugar, los modelos de regulación tarifaria que buscan equidad distributiva median entre asegurar una ganancia razonable de largo plazo para las empresas prestadoras y que la tarifa permita la ampliación sostenida del acceso al servicio público. El aspecto de la ganancia razonable fue largamente debatido y analizado por múltiples autores (Armstrong, Cowan y Vickers, 1994), y a grandes rasgos pretende reflejar en la tarifa el costo medio del servicio más un porcentaje que representa su rentabilidad, y que debería ser suficiente para sostener la calidad de prestación del servicio, extender la cobertura del sistema, y generar un excedente para ser apropiado por las empresas. Bajo este modelo que aspira a lograr equidad distributiva, existe una mayor preponderancia de la intervención pública en la regulación (sea a través de empresas estatales o entes reguladores) y subyace el supuesto de que la prestación de un servicio público no es necesariamente una mer- 
cancía igual a otras que se comercializan, sino que debe aspirar a universalizar la cobertura. Bajo este modelo, la fijación de la tarifa es un instrumento para asegurar esa expansión, al tiempo que debe permitir una ganancia razonable de largo plazo para las empresas.

Más allá de los múltiples matices y mediaciones existentes entre ambos modelos aquí presentados de forma típica-ideal, y de las particularidades que ha adquirido el proceso durante su instauración, la historia institucional reciente de regulación tarifaria de los servicios públicos en Argentina estuvo signada por marchas y contramarchas muy marcadas entre ambos modelos. El caso del gas natural por redes no es una excepción.

\section{REGULACIÓN POR MONOPOLIO ESTATAL (1967-1991)}

La organización del servicio público de transporte y distribución de gas natural por redes en Argentina era ejercida monopólicamente por Gas del Estado, empresa estatal que adquiría de Yacimientos Petrolíferos Fiscales (YPF) el gas natural en boca de pozo, lo trataba para poder inyectarlo en los gasoductos troncales y los distribuía a los usuarios finales. Gas del Estado era la única empresa que podía importar gas natural y distribuir gas licuado de petróleo. Bajo este modelo de monopolio estatal, la participación del sector privado era marginal y se daba, principalmente, a través de tres mecanismos: $a$ ) tareas menores de mantenimiento cedidas en la década de 1970 a través de la llamada privatización periférica de YPF (Castellani y Serrani, 2010); b) participación en ciertas obras de infraestructura energética como la construcción y mantenimiento de gasoductos, y c) el transporte por esos gasoductos construidos por agentes privados a cambio de un margen de transporte, ya que la propiedad del fluido era de Gas del Estado (Fundación de Investigaciones Económicas Latinoamericanas, 1987).

El cuadro tarifario de la demanda final estaba compuesto por un cargo fijo y por una tarifa por metro cúbico consumido, política que se mantuvo a partir de la privatización de Gas del Estado hasta la actualidad. Ante el incremento del consumo, se realiza una primera diferenciación por localización de consumos no domésticos. Se presentan nueve zonas de acuerdo con diferencias de costo por transporte según las distancias existentes entre los yacimientos y los centros de consumo, alentando que la actividad industrial se sitúe en lugares cercanos a las zonas de captación. En 1985 se incorpora una diferenciación por localización para consumos domésticos, con cinco zonas que contemplaba en la tarifa final la distancia existente entre los yacimientos y los centros de consumo, las condiciones climáticas, y el grado de desarrollo económico. En este sentido, si bien el cargo fijo era uno en todo el país, se incorporaron tarifas crecientes para las escalas de mayores consumos, a modo de incentivar el uso racional del recurso por la vía tarifaria.

Respecto a la regulación tarifaria, durante la etapa final de la industrialización por sustitución de importaciones (1967-1975), y con base en los datos disponibles, la política de precios estuvo orientada por criterios de política pública con aspiraciones de equidad distributiva antes que de eficiencia económica. En este subperiodo, el precio de transferencia de YPF a Gas del Estado presenta una sistemática caída, que es mayor a la de la tarifa media del gas distribuido por Gas del Estado. De esta manera, se buscaba generar incentivos a la demanda final y cuidar las finanzas de Gas del Estado, ya que el precio de transferencia fue cinco veces superior al precio de adquisición (véase gráfica 1). Con el inicio de la dictadura cívico-militar en 1976, y ante la búsqueda de adecuar los precios internos a la evolución de los precios internacionales, la tendencia se revierte y el margen para Gas del Estado comienza a ser cada vez menor (véase gráfica 1), producto tanto del encarecimiento del precio de gas importado de Bolivia como del precio de transferencia por las políticas 
antiinflacionarias y cambiaria que provocaron simultáneamente un retraso en el ajuste de las tarifas y de la tasa de cambio (Kozulj y Pistonessi, 1989, p. 124). De este modo, hacia 1983 el costo de adquisición más que se duplica al promedio del periodo anterior, por lo que llega a ser más caro que la tarifa de distribución, redundando en una crítica situación económico-financiera de Gas del Estado, que se vio agravada por su endeudamiento. Finalmente, con el regreso de la democracia se recompone la relación entre el costo de adquisición y el precio de venta, aunque desacoplado de la dinámica internacional. En un contexto de alta inflación como fue toda la década de 1980, con la lenta recomposición del margen de distribución el gobierno buscó contener la constante escalada de los precios del resto de la economía (Kozulj y Pistonessi, 1989, p. 124).

La evolución de las tarifas finales se ajusta a la dinámica de los tres subperiodos presentados anteriormente: mientras bajo un modelo de protección industrial la evolución de la tarifa industrial fue decreciente y menor en términos reales que la residencial, se equiparan hacia finales de ese periodo. A partir de 1977, no sólo se desacopla la dinámica industrial de la residencial -al ser significativamente mayor hasta 1980-, sino que se agrega una nueva categoría tarifaria para los altos consumos residenciales (que pasa a ser considerablemente más alta), lo que traslada mayores costos a los usuarios de mayor consumo.

Si bien esta tendencia comienza a cambiar hacia finales de la dictadura -donde caen los precios industriales y se equiparan con los residenciales de altos consumo-, la dinámica se mantendría durante toda la presidencia de Raúl Alfonsín, periodo en el cual, tras la puesta en operación del megayacimiento gasífero Loma La Lata, en 1977, y el desarrollo del gasoducto Centro-Oeste, en 1981, se incentiva, mediante tarifas residenciales decrecientes, la utilización de gas natural para sustituir el uso de otros combustibles líquidos, más caros y menos eficientes en términos energéticos: "No cabe duda que la cuestión de la equidad social se relaciona de manera más significativa con las posibilidades de acceso al consumo de gas natural por parte de las familias de menores recursos que con el nivel relativo de la tarifa. Esto al menos en tanto que la tarifa de gas distribuido ha tenido en Argentina una relativa baja incidencia en los presupuestos familiares" (Kozulj y Pistonessi, 1989, p. 134) (véase gráfica 2).

En definitiva, y aun con diferencias sustantivas en los gobiernos que las pusieron en práctica entre 1967-1991, el modelo regulatorio buscó utilizar la política tarifaria con fines antiinflacionarios, al impedir trasladar la totalidad del costo de producción a los usuarios finales y de buscar, a la vez, sustituir consumos de combustibles líquidos por un incremento de la utilización residencial de gas natural. Este esquema de monopolio natural bajo el control del Estado a través de Gas del Estado fue transformado en 1992, cuando se fracciona la empresa en múltiples unidades de gestión y se transfieren a empresas privadas, generándose un nuevo modelo regulatorio para el sector.

\section{Modelo DE EFICIENCIA ECONÓMICA MARGINALISTA (1992-2001)}

Con la renuncia del gobierno radical de Raúl Alfonsín, azorado por la crisis económica, política, social e hiperinflacionaria, y el llamado adelantado a elecciones, en junio de 1989 asume la presidencia Carlos Menem. Aun implementando prontamente el decálogo de trasformaciones del Consenso de Washington, los primeros dos años de gestión estuvieron definidos por la hiperinflación, la crisis económica, y las disputas en el campo laboral-sindical por el avance de la desregulación del mercado de trabajo y la amplia privatización de empresas estatales (Pucciarelli, 2011). 


\section{GRÁFICA 1. COSTO MEDIO DE ADQUISICIÓN DEL GAS NATURAL PARA GAS DEL ESTADO, TARIFA MEDIA DE GAS DISTRIBUIDO SIN IMPUESTOS Y RELACIÓN PORGENTUAL. ARGENTINA, 1965-1989 (EN PESOS ARGENTINOS DE 1979 POR METRO CÚBICO)}

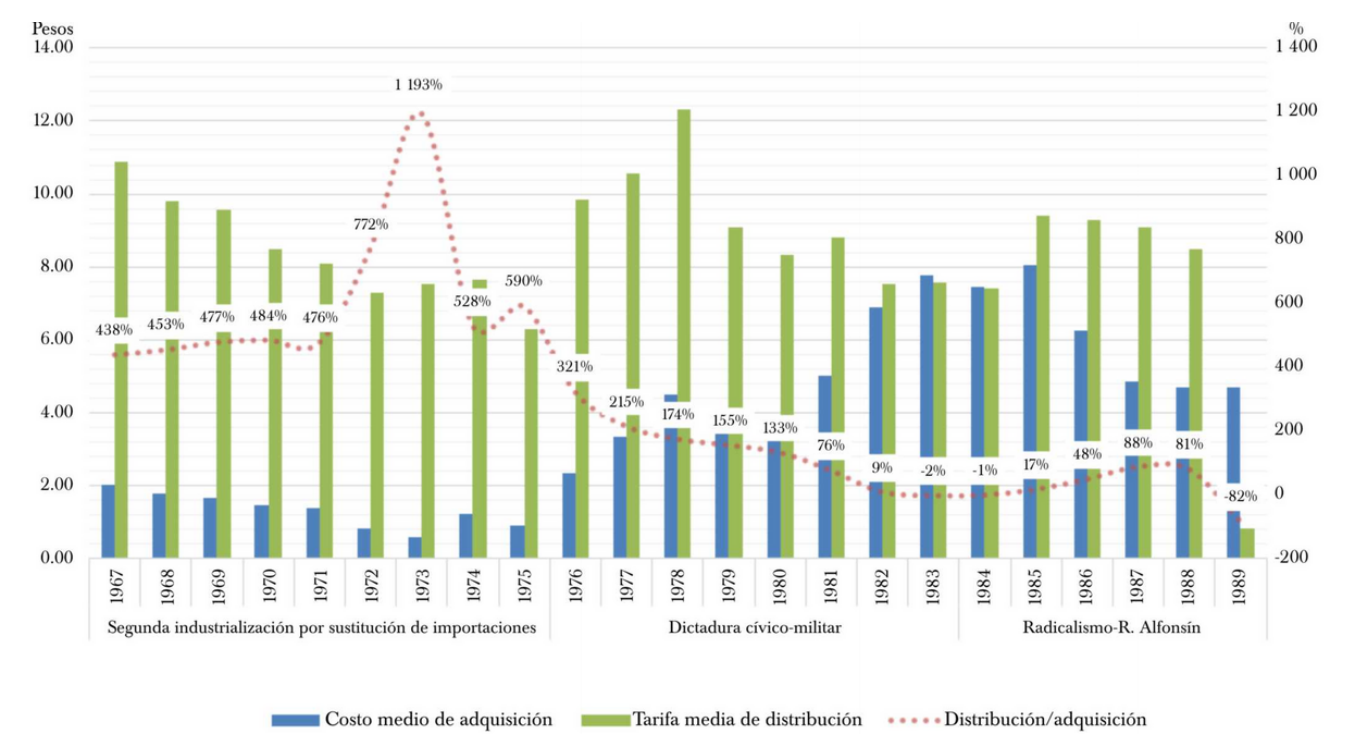

Fuente: elaboración propia con base en Kozulj y Pistonessi (1989).

En términos retóricos, las reformas neoliberales en el sector energético buscaron remover la regulación estatal para permitir que los mecanismos competitivos de mercado y la libre entrada de oferentes privados sea la estructura institucional que asigne los bienes y servicios. En el marco del servicio público de gas natural por redes, se desarticuló el monopolio estatal al privatizar Gas del Estado, subdividiéndola en dos sociedades transportistas y ocho de distribución monopólicas (en 1997 se adicionaría una nueva concesionaria, Gasnea). Se introdujeron límites en la propiedad del capital de las compañías privadas de la cadena gasífera impidiendo la integración vertical, se eliminaron los subsidios cruzados y, de hecho, los controles estatales sobre los compromisos de inversión.

El entramado normativo para la regulación del servicio se organizó a partir de la Ley 24.076 del 12 de junio de 1992 y su decreto reglamentario 1.738 del 28 de septiembre del mismo año, las reglas básicas y el reglamento del servicio de la licencia de distribución y sus normas complementarias y concordantes (marco regulatorio del gas). Bajo este esquema se constituyó un organismo autárquico regulador del servicio, el ENARGAS, que tuvo por función reflejar en las tarifas una rentabilidad razonable para las empresas, teniendo expresas restricciones para que las tarifas finales no sean menores al costo marginal del servicio. Siguiendo la estructura diseñada bajo el modelo de monopolio estatal de Gas del Estado, la tarifa es el resultado de la suma de tres componentes: a) el precio del gas en el punto de ingreso al sistema de transporte; $b$ ) el costo de transporte, y $c$ ) el margen de distribución (a lo que se suman otros cargos extratarifarios y los impuestos).

Siguiendo lo definido en el marco regulatorio del gas y lo analizado en Serrani (2018), el nuevo régimen tarifario dispuso simultáneamente dos sistemas que modificaron la asignación del modelo anterior. 


\section{GRÁFICA 2. EVOLUCIÓN DEL PRECIO FINAL DE LAS TARIFAS PARA USUARIOS DE CONSUMO BAJO Y ALTO, Y DEL SECTOR INDUSTRIAL. ARGENTINA, 1965-1989. AÑO BASE $1970=100$}

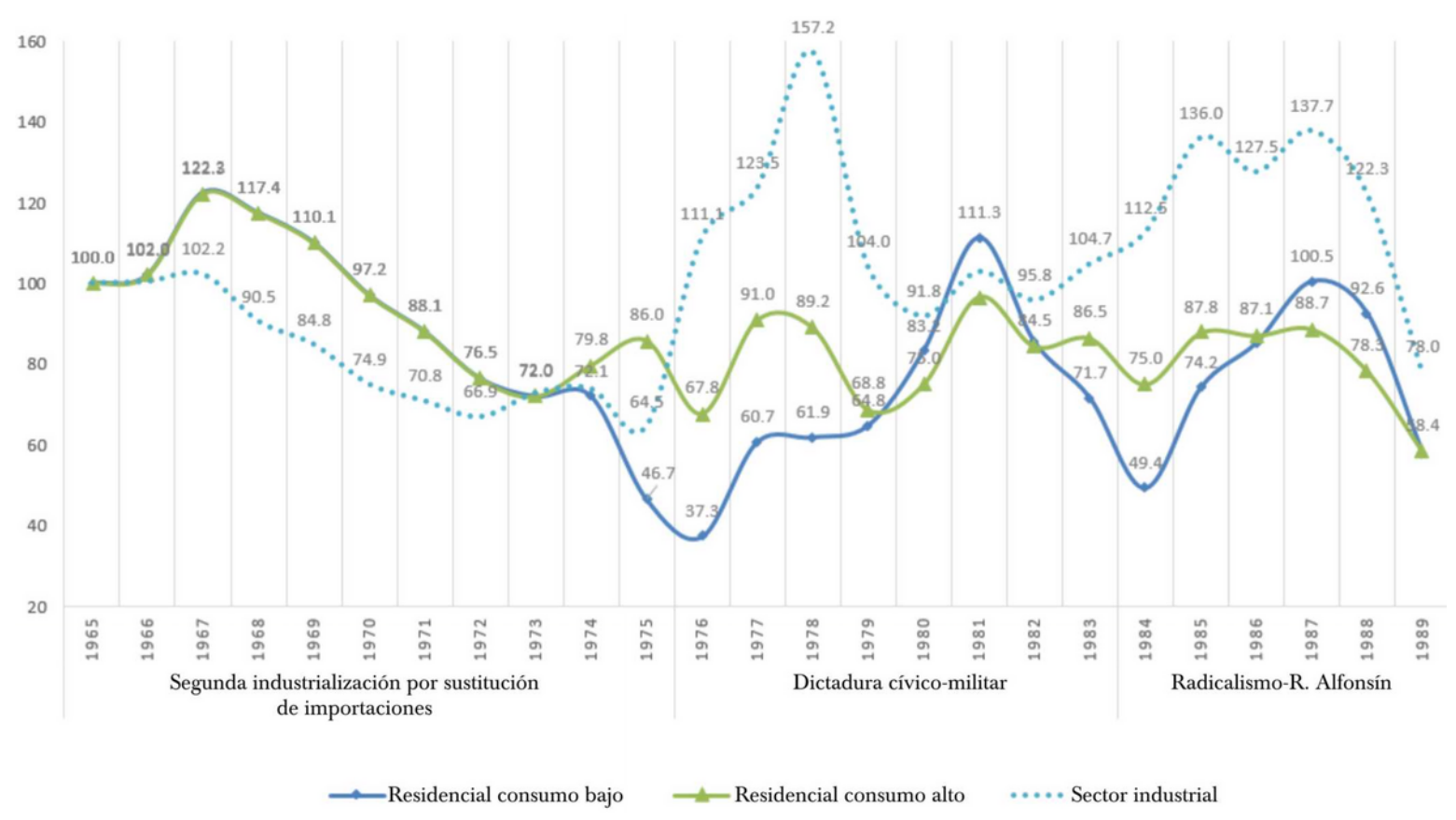

Fuente: elaboración propia con base en Kozulj y Pistonessi (1989).

El primero es el sistema de pass-through, que habilita a las empresas distribuidoras a reflejar en la tarifa el precio desregulado que paga por el gas natural que adquiere luego de la negociación con los productores en el punto de ingreso al sistema de transporte. La desregulación en la fijación del precio del gas natural entre productores y distribuidores es la principal diferencia con el modelo de monopolio estatal. Anteriormente, este era pactado entre las dos empresas estatales, ya que yPF le vendía a Gas del Estado a un precio regulado y fijado estatalmente, y no siempre era superior a su costo de extracción. Respecto al costo de transporte que las distribuidoras pagan a las transportistas por llevar el gas natural desde el punto de ingreso al sistema de transporte hasta la puerta de entrada al sistema de concesión de distribución, este es regulado y fijado por el EnARGAS, y las distribuidoras también lo trasladan directamente a los usuarios finales. En síntesis, bajo este sistema las empresas de distribución no obtienen ganancia alguna por el precio de gas natural ni por el costo de transporte que les cobra a los usuarios finales.

El segundo es el sistema de price-caf $2^{2}$, donde el EnARGAs quedó encargado por el marco regulatorio de fijar precios máximos para las tarifas, para un periodo durante el cual las tarifas podían ser ajustadas por la evolución del índice de precios. Este modelo procura reflejar en la tarifa final la evolución de los costos de las empresas de transporte y distribución, los ocasionados por la pres-

\footnotetext{
${ }^{2}$ Stephen Littlechild (1983) diseñó el price-cap cuando, de un informe presentado al gobierno del Reino Unido a comienzos de 1980, lo propuso como novedosa metodología para la regulación tarifaria del incipiente proceso de privatización de empresas públicas.
} 
tación del servicio y la obtención de una ganancia razonable. El sistema de price-cap busca que las empresas optimicen sus costos para que el precio final sea más bajo que el precio máximo fijado por el regulador, y así competir con otros oferentes del servicio para obtener una mayor cuota de mercado. De esta manera, el concesionario ganaría por la ampliación de la cobertura de su servicio y los usuarios finales, por una tarifa más baja. Sin embargo, cuando a un monopolio natural se le aplica la lógica del price-cap, son pocos los incentivos que se generan para reducir los costos del servicio, bajar la tarifa final, e incrementar por eficiencia la tasa de rentabilidad, ya que las concesionarias no podían competir por la obtención de nuevos usuarios fuera de su área de concesión, lo que implicó, de hecho, que la reducción de costos y/o el aumento de productividad redundara en un incremento de la rentabilidad para las empresas privatizadas de servicios públicos.

Asimismo, el marco regulatorio también disponía que las tarifas debían ajustarse semestralmente por una variación de: $a$ ) los indicadores del mercado internacional (price producer index); $b$ ) por variaciones en el precio del gas comprado, y $c$ ) por variaciones en el costo del transporte (Serrani, 2018). Asimismo, la revisión semestral del índice de precios al productor debía ser ajustado por un factor de eficiencia $X$ y un factor de inversión $K$, y las tarifas debían revisarse cada cinco años $\mathrm{S}^{3}$ Por otra parte, las tarifas de transporte y distribución de gas se calculaban en dólares estadunidenses, aunque los cuadros tarifarios publicados los expresaban en pesos convertibles a la paridad establecida en el decreto 2.128/91 del 17 de octubre de 1991, que reglamentó la Ley 23.928 del 28 de marzo del mismo año.

En efecto, después de las reformas estructurales, el modelo regulatorio buscó la eficiencia económica del sistema, por lo que la totalidad del costo marginal de sus componentes fueron trasladados a la tarifa. Es por ello que en el periodo 1992-2001 4 en promedio, las tarifas finales fueron $221 \%$ superiores al costo del gas natural en boca de pozo, o lo que es lo mismo, el costo de gas en la tarifa final representó tan sólo $31 \%$ en promedio en ese periodo (véase gráfica 3 ). Al analizar la evolución de las tarifas residenciales, el modelo sólo logró bajar la tarifa promedio residencial $4.1 \%$ entre 1996 y 1998, y tuvo que esperar a 2001 para recuperar el valor máximo de la década (1996). Sin embargo, cuando se analiza entre puntas, la tarifa se incrementó $9.9 \%$ en dólares bajo la paridad cambiaria, mientras que el gas natural en boca de pozo casi triplicó el incremento (36\%), esto a pesar de un contexto general de crisis macroeconómica y de una caída de la actividad industrial y de los precios (Basualdo, 2006).

El modelo de mercado trasladó el costo marginal de los componentes tarifarios a los usuarios finales, y al no disponer de tarifas que discriminaran según los diferentes segmentos de consumo, generó un patrón regresivo en cuanto al valor final del metro cúbico consumido. Mientras un usuario de alto consumo abonó 3.9 dólares por millón de втц 5 consumido en promedio, un usuario de consumo medio pagó 4.6 dólares por millón de BTU, es decir $17.7 \%$ más por la misma unidad calórica (véase gráfica 3). A menores niveles de consumo, el peso del cargo fijo que se abonaba por factura tiene un peso mayor para los usuarios que menos consumen. De tal manera que un usuario de bajo consumo pagó en promedio 6.2 dólares por millón de BTU, un precio $33 \%$ mayor al de un usuario de consumo medio, pero $56.6 \%$ más que uno de alto consumo. Finalmente, para comprender la dinámica del modelo regulatorio y de las tarifas residenciales, se las vinculó con

\footnotetext{
${ }^{3}$ Algo que sólo sucedería en el primer lustro desde la privatización.

${ }^{4}$ Aunque en las elecciones de 1999 cambió el partido en el gobierno (luego de la reelección de Carlos Menem en 1995), la política energética del gobierno de la Alianza, liderado por Fernando de la Rúa, continuó la orientación del marco regulatorio aprobado en 1992.

${ }^{5}$ British thermal unit (unidad térmica inglesa).
} 
GRÁFICA 3. TARIFAS PARA USUARIOS DE CONSUMOS BAJO, MEDIO Y ALTO, PRECIO DEL GAS NATURAL EN BOCA DE POZO Y DEL SALARIO. ARGENTINA, 1992-2001 (EN DÓLARES POR MILLONES DE BTU Y DÓLARES CORRIENTES)

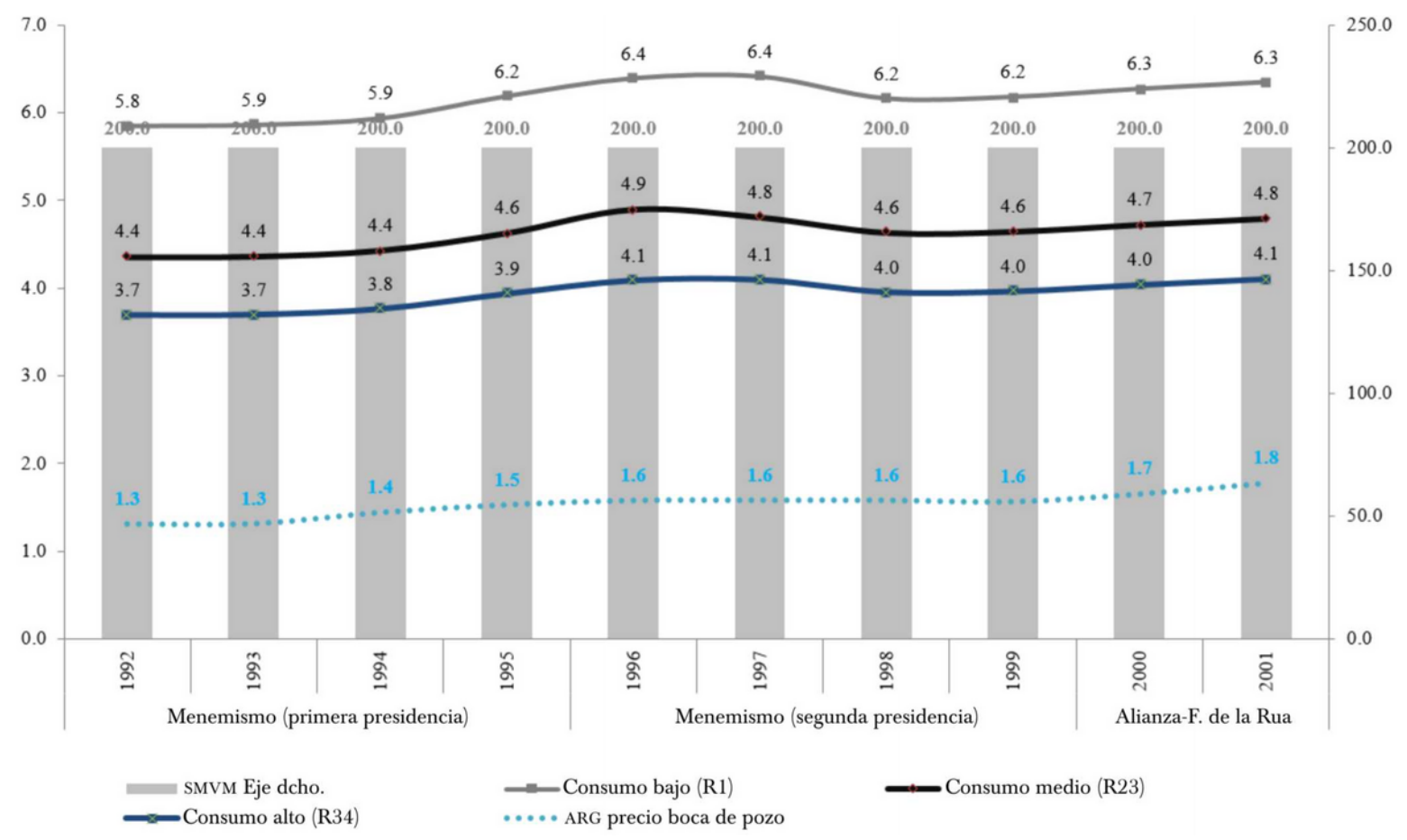

Fuentes: elaboración propia con base en Ente Nacional Regulador del Gas. Recuperado de https://www.enargas.gob.ar; Ministerio de Energía y Minería. Recuperado de https://www.argentina.gob.ar/energia, y Ministerio de Trabajo, Empleo y Seguridad Social. Recuperado de https://www.argentina.gob.ar/trabajo años 1992-2001.

la evolución del salario en dólares, que toma como unidad de medida el salario mínimo, vital y móvil (salario mínimo). Así, se puede apreciar que el salario mínimo estuvo congelado durante toda la década de 1990 mientras que las tarifas aumentaron en promedio $9.9 \%$, lo que generó un proceso de erosión en los ingresos de los sectores más postergados.

Sin embargo, esta particular evolución tarifaria no hubiese sido posible sin lo sucedido dos años antes de la privatización de Gas del Estado en 1992: entre marzo de 1991 y enero de 1992, el valor del cargo variable residencial incrementó $64.5 \%$ en pesos convertibles, y si toma la evolución respecto a enero de 1993 (primer mes completo de inicio de las nuevas concesiones privadas), el incremento fue de $101.2 \%$ (FLACSO, 1998, p. 34). Esto implicó un incremento sobre la tarifa promedio residencial de $58.1 \%$ para enero de 1993 . Es decir que, al momento de inicio del funcionamiento de las concesiones privadas, los aumentos tarifarios más significativos ya habían sido efectuados, siendo los costos asociados atribuidos a la gestión de la empresa estatal Gas del Estado. 
En definitiva, el modelo regulatorio no tuvo la necesidad de generar incentivos para bajar costos que permitieran tener prioridad de despacho, ni hubo competencia de mercado a través de los precios de la energía para ampliar la cobertura ni ganar nuevos clientes, ya que la diagramación institucional del servicio de distribución consolidó mercados monopólicos (naturales) distribuidos en regionales.

\section{Modelo De EQUidAD DistRibutiva (2002-2015)}

Tras la crisis de finales de 2001, el 7 de enero de 2002 se sanciona la Ley 25.561 de emergencia pública y de Reforma del Régimen Cambiario que declara la emergencia social, económica, administrativa, financiera y cambiaria. Bajo esta ley quedaron sin efecto todas las cláusulas de ajuste en dólares de las tarifas y cualquier otro tipo de cláusula indexatoria para la economía en su conjunto. De esta manera, los precios y tarifas quedaron establecidos en pesos a la paridad de cambio un peso igual un dólar. Asimismo, instó al poder ejecutivo nacional a renegociar los contratos de los servicios públicos, tomando en consideración el impacto de las tarifas en la competitividad de la economía, en la distribución de los ingresos, y en la rentabilidad de las empresas. En 2003, el presidente Néstor Kirchner crea la Unidad de Renegociación y Análisis de Contratos de Servicios Públicos, la cual quedó encargada de la renegociación contractual con las empresas privatizadas.

\section{Transición y congelamiento tarifario (2002-2011)}

En medio de una crisis social estructural que se desarrolló durante toda la presidencia interina de Eduardo Duhalde (2002-2003), y de mucha conflictividad económica derivada de la pesificación asimétrica de la economía (Basualdo, 2006), dentro de la cual se encontraba la pesificación de las tarifas energéticas, bajo la presidencia de Néstor Kirchner (2003-2007), el ministro de Planificación tuvo como misión dar comienzo a la renegociación contractual, en el marco de la unidad de renegociación, con la firma de acuerdos transitorios, en primer lugar, y, luego, de actasacuerdo de renegociación contractual integral. Estos buscaban fijar nuevos criterios para dar por finalizado el proceso de transición tarifaria que, sin embargo, se extendería de 2004 y a 2014.

Después de la pesificación tarifaria, el modelo regulatorio que emergió para el periodo de transición se organizó alrededor de cuatro transformaciones que marcaron un quiebre con el modelo promercado de la etapa anterior (Serrani, 2018): a) se fijó un régimen tarifario de transición -con readecuación de precios y tarifas, incluidas variaciones del precio del gas en el punto de ingreso al sistema de transporte, la tarifa de transporte y la de distribución-; b) al anularse la indexación semestral por el índice de precios al productor (inflación de Estados Unidos) se estableció un mecanismo de monitoreo de costos no automático para ajustar semestralmente las tarifas finales; $c$ ) se fijó un régimen de tarifa social destinado a hogares de bajos ingresos (no se implementó hasta 2016), y $d$ ) se determinó la necesidad de realizar una revisión tarifaria integral para fijar un nuevo régimen de tarifas máximas (price-cap) para los próximos cinco años.

Hasta llegar a la revisión tarifaria integral, para el periodo de transición se había fijado una nueva tarifa de transición asociada a un mecanismo de monitoreo de costos de actualización semestral, para que, en un contexto inflacionario, la variación de la estructura de costos de las empresas se viera reflejado en la evolución de las tarifas. Sin embargo, luego de la firma de las actas transitorias, y a medida que la renegociación por la revisión tarifaria integral se extendía en el tiempo -sin que se implementara el mecanismo de monitoreo de costos ni se publicaran nuevas tarifas 
de transición-, se firmaron actas-acuerdo finales, lo que ratificó lo establecido en el primer acuerdo transitorio. Este proceso se extendió entre 2006 y 2014, y sólo Gas Natural Fenosa recibió, en 2007, un incremento en su tarifa de distribución.

La renegociación de las licencias y la regulación tarifaria atravesarían dos presidencias, la de Néstor Kirchner (2003-2007) y la primera de Cristina Fernández de Kirchner (2007-2011), y se dio un contexto de caída de la extracción local (ininterrumpida entre 2004 y 2011) y de ampliación de la demanda global del sistema gasífero, brecha que paulatinamente fue creciendo y que la gestión energética del ministro Julio De Vido cubrió con importaciones energéticas. Con el propósito de financiar las importaciones energéticas, desde el Ministerio de Planificación se diseñó un cargo tarifario para todos los usuarios de gas natural, denominado cargo al gas importado, monto que las distribuidoras que lo recaudaban depositarían en un fideicomiso y que el Estado usaría para pagar las importaciones. Sin embargo, la aplicación del cargo fue judicializado por el impacto en las tarifas, dejando sin efecto su aplicación. A los pocos meses el cargo se modificó y sólo lo pagaban los usuarios residenciales y comerciales de alto consumo (y algunas industriales y centrales eléctricas). La aplicación del cargo tuvo un bajo impacto, ya que lo recaudado sólo cubrió una mínima parte de las divisas necesarias para garantizar las crecientes importaciones de gas natural. La agresiva política de importación terminaría agravando problemas estructurales para la economía nacional, ya que las importaciones llegaron a representar $15.2 \%$ del consumo anual en 2011 (casi un tercio de la demanda en el pico de consumo de invierno), y destinando 2357 millones de dólares de subsidios para mantener el diferencial entre los precios de importación de gas natural y los de venta interna, significativamente más baratos.

\section{Transición e incremento tarifario (2012-2015)}

En el inicio de la segunda presidencia de Cristina Fernández de Kirchner (2011-2015) se produce un cambio en la gestión de la política económica, que sucede desde el Ministerio de Planificación hacia el de Economía, liderado por Axel Kicillof, quien encaró una política de sintonía fina de la economía, al buscar destrabar problemas estructurales para volver a crecer en el mediano plazo. En el marco más general de caída de la producción gasífera, déficit de la balanza comercial energética, e incremento de subsidios al gas natural (Serrani y Barrera, 2018), el gobierno nacional expropia 51 \% de YPF, la petrolera más grande del país, privatizada en 1993 y traspasada a Repsol en 1999.

En este contexto de búsqueda de sintonía fina y de recuperación de yPF para incrementar la extracción local (entre otros objetivos), en el servicio residencial de gas natural se buscó mejorar la tasa de rentabilidad de las empresas ante el incumplimiento de la sanción de nuevos cuadros con tarifas de transición, la implementación del mecanismo de monitoreo de costos, y la revisión tarifaria integral. En 2012 se creó un fideicomiso denominado Fondo para Obras de Consolidación y Expansión de la Red de Distribución de Gas (Focegas), el cual sería financiado por un cargo fijo que todos los usuarios abonarían y cuyo monto serviría para que las distribuidoras financien sus planes de inversiones.

Posteriormente, en 2014 se fijó la primera modificación en todos los cuadros tarifarios de distribución de gas natural desde 2004: se incrementó el precio del gas en el punto de ingreso al sistema de transporte ( $550 \%$ en promedio) y el costo del transporte $(20 \%)$, los cuales fueron trasladados al sistema pass-through a la tarifa final. También se incrementó el margen de distribución de forma diferencial para cada empresa. 
Al comparar los efectos de los distintos modelos regulatorios en las tarifas -en el preludio de la convertibilidad cambiaria, la privatización de Gas del Estado y el inicio de las concesiones privadas de distribución-, el valor de las tarifas finales residenciales tuvo un incremento de $58.1 \%$ entre marzo de 1991 y enero de 1993, mientras que, con la ley de emergencia pública, la devaluación y la pesificación tarifaria de 2002, este presentó una caída en dólares de 58.2 \% (véase gráfica 4). Es decir, un efecto de la misma magnitud, pero en sentido inverso a la del periodo señalado anteriormente.

Desde 2002 hasta 2013 el nuevo modelo regulatorio de equidad distributiva mostró una dinámica distinta entre precios del gas, tarifas y salarios. Mientras el gas en boca de pozo creció $193.5 \%$ (de un dólar por millón de BTU en 2002 a 2.9 en 2013), las tarifas se contrajeron $56.9 \%$ para usuarios de consumo bajo y medio, pero crecieron $50.5 \%$ para los de alto consumo por efecto del cargo por gas importado, y el salario mínimo tuvo un incremento en dólares de $560.1 \%$. Esta dinámica implicó una fuerte caída de la porción del salario necesaria para afrontar el pago del servicio, siendo un mecanismo de mejora indirecta de los ingresos de los usuarios finales, al tiempo que la tarifa abonada por los usuarios residenciales de consumo bajo y medio terminó siendo más barata que el gas natural en boca de pozo (prohibido por el marco regulatorio del gas), brecha que fue cubierta con subsidios estatales.

Con las nuevas tarifas de 2014, estas se incrementaron poco a poco para usuarios de consumo bajo (17.9\%), medio $(44.8 \%)$ y alto $(57.2 \%)$, de forma que la reducción de subsidios fuera más fuerte en los usuarios de mayor consumo, ya que se buscó proteger a aquellos que, por tamaño de los hogares y por prácticas de ahorro, logran un uso menos intensivo del servicio público. Al mismo tiempo, el salario mínimo se redujo en dólares casi 14 \% como efecto de la devaluación de $48 \%$ en 2014 respecto al año anterior (véase gráfica 4).

La combinación de la política de pesificación tarifaria y administración de los precios de la economía de este modelo regulatorio implicó una redistribución del ingreso en favor de los usuarios finales. Si en 1992 un usuario de consumo medio, por una factura sin impuestos de 180 metros cúbicos mensuales abonaba 29 dólares, obligado a destinar $14.5 \%$ de un salario mínimo, hacia finales de la convertibilidad esos valores casi no se habían modificado en dólares corrientes (32 dólares y $15.9 \%$ del salario). Luego de la pesificación y devaluación de 2002 , por el mismo consumo hubiese pagado trece dólares, 18.5 \% de un salario mínimo, y para finales del kirchnerismo, luego de los aumentos en las tarifas de transición y con la devaluación de 2014, esa factura pasó a valer once dólares, $2 \%$ del salario.

Sin embargo, en 2014 un conjunto de medidas cautelares impidió el cobro pleno de las nuevas tarifas de transición, lo que generó un mapa caótico de tarifas gasíferas en el país (Serrani, 2018). En este contexto, y bajo una fuerte presión de las empresas para recomponer sus ingresos, en 2015 el Estado transfirió 2590 millones de pesos a las distribuidoras, con el propósito de que no se cortara la cadena de pago del sector.

En efecto, después de la devaluación de 2002 y a la pesificación de tarifas se constituyó híbridamente un modelo regulatorio que, sin desandar el entramado normativo sancionado en la década anterior, buscó alcanzar equidad distributiva antes que la eficiencia marginalista del sistema.

Sin embargo, resulta necesario destacar algunos matices interpretativos sobre la anterior definición y los efectos que tuvo sobre el sector. La ampliación de la cobertura (la incorporación de 587335 usuarios residenciales entre 2002 y 2015 incrementó $40 \%$ la cobertura) se dio por una nueva orientación de la política pública, que desarticuló el modelo tarifario basado en el costo marginal por uno fundado en tarifas de transición reguladas estatalmente. Sin embargo, esta orien- 
GRÁFICA 4. TARIFAS PARA USUARIOS DE CONSUMOS BAJO, MEDIO Y ALTO, DEL PRECIO DEL GAS NATURAL EN BOCA DE POZO Y DEL SALARIO. ARGENTINA, 2001-2015. EN DÓLARES POR MILLONES DE BTU Y DÓLARES CORRIENTES

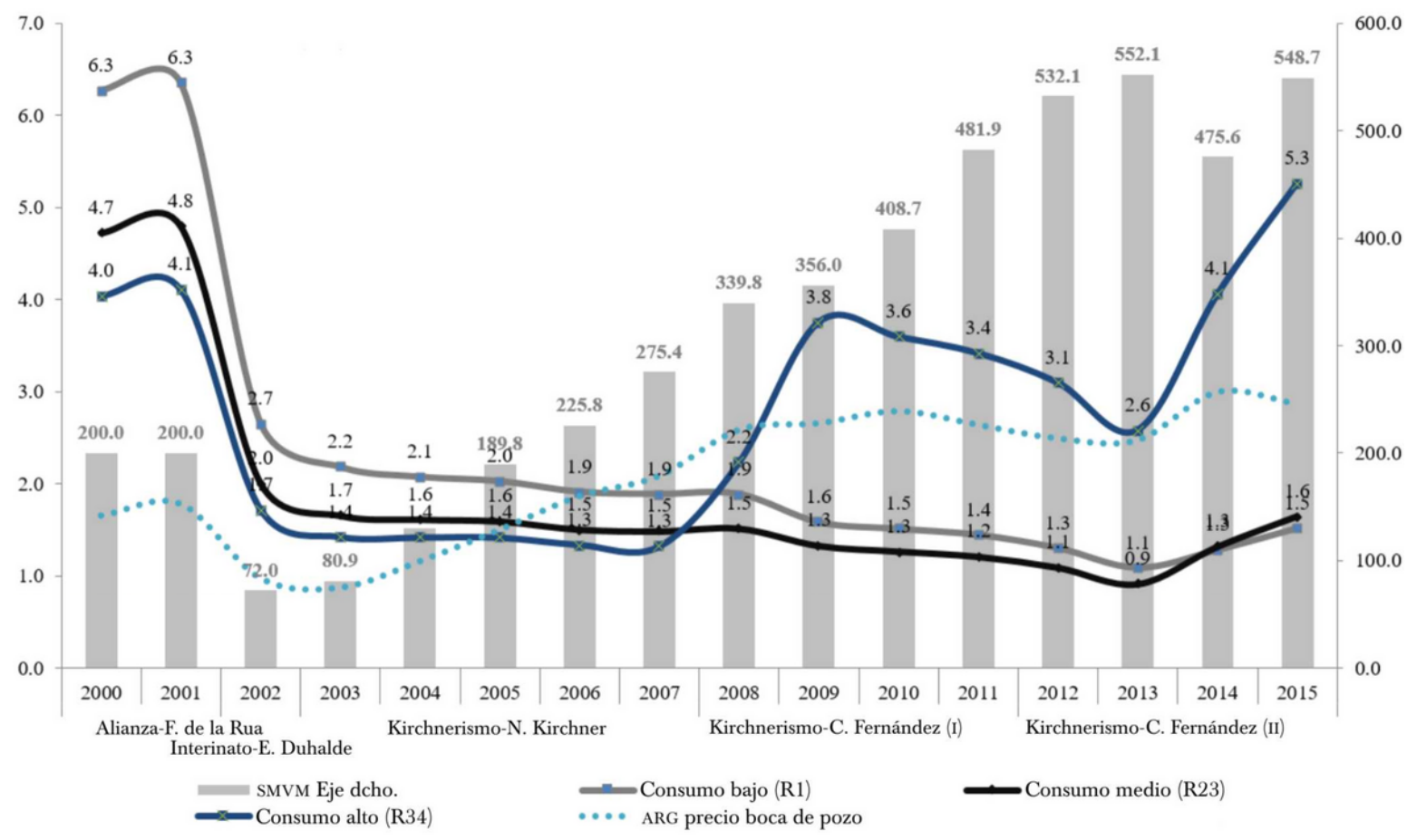

Fuentes: elaboración propia con base en Ente Nacional Regulador del Gas. Recuperado de https://www.enargas.gob.ar; Ministerio de Energía y Minería. Recuperado de https://www.argentina.gob.ar/energia y Ministerio de Trabajo, Empleo y Seguridad Social. Recuperado de https:/www.argentina.gob.ar/trabajo años 2000-2015.

tación de la política tarifaria deterioró los ingresos de las distribuidoras a la vez que aumentó los subsidios energéticos, teniendo efectos distorsivos para la economía nacional (Barrera y Serrani, 2018), evidentes durante el segundo mandato de Cristina Fernández de Kirchner (2011-2015). Y al no haberse transformado el marco regulatorio del gas, al nuevo gobierno de corte neoliberal le resultó sumamente sencillo volver al modelo de la década de 1990.

\section{RETORNO AL MODELO DE EFICIENCIA MARGINALISTA (2016-2017)}

En diciembre de 2015 se produce en Argentina una nueva alternancia en el gobierno, luego de tres mandatos consecutivos del Frente para la Victoria bajo las presidencias de Néstor Kirchner y dos periodos de Cristina Fernández de Kirchner. Con la llegada al poder ejecutivo de la Alianza Cambiemos se lanzó un programa económico neoliberal para sincerar los precios con una reducción escalonada de subsidios, que para 2015 representaban $4.1 \%$ del PIB, siendo los destinados a la energía los más relevantes, con $2.9 \%$ (Muras et al., 2015, p. 8). 
Para avanzar en la revisión tarifaria integral, el gobierno disolvió la Unidad de Renegociación y concentró toda la responsabilidad en el naciente Ministerio de Energía y Minería, liderado por Juan José Aranguren, exdirector de Shell en Argentina y directivo de la empresa por tres décadas. En los primeros meses de 2016, las distribuidoras firmaron nuevos acuerdos transitorios, obteniendo un nuevo régimen de tarifas de transición, derogando el cargo por gas importando y pasando el Focegas a la tarifa (Resolución 28/2016, 1 de abril). De esta manera, buscó reducir los subsidios al gas natural (diferencial entre el precio de importación y el de venta local) con incrementos semestrales del precio en el punto de ingreso al sistema de transporte hasta 2019, además de sancionar nuevos cuadros tarifarios de transición con incrementos para mejorar los ingresos de transportistas y distribuidoras.

Sin embargo, ante la magnitud de los incrementos tarifarios planteados y la descoordinación y apuro por parte del Ministerio de Energía y Minería en su instauración, una gran cantidad de recursos de amparo detuvieron la aplicación de las nuevas tarifas. En junio de 2016, y ante el desconcierto del Ministerio de Energía y Minería respecto a los recursos de amparo, se pusieron límites porcentuales a los incrementos en las facturas finales (400\% para residenciales respecto del valor del metro cúbico consumido en la factura del mismo periodo del año anterior). Sin embargo, estos topes eran más altos a los de los cuadros tarifarios de transición. Esta situación llevó a que un mes más tarde se sancionara una nueva resolución con topes porcentuales en el valor de la factura final respecto a la misma del año anterior, independientemente de cuál había sido su consumo (Resolución 99/2016, 7 de junio). En septiembre de 2016, el Ministerio de Energía y Minería, por expreso pedido de la Corte Suprema de Justicia, llevó adelante una audiencia pública para tratar su propuesta. A pesar de la extendida participación de expertos, académicos y diversos grupos de interés de la sociedad civil, la propuesta original de reducción de subsidios e incremento tarifario se mantuvo inalterable, y el ministro de Energía y Minería instruyó al EnARGAS que llevara adelante los acuerdos necesarios para finalizar la revisión tarifaria integral.

Así, se estableció un sendero de precios para el gas natural, con el fin de incrementar el precio promedio de 1.29 dólares por millón de BTU de inicios de 2016 hasta llegar a 6.78 en octubre de 2019 (incremento de $427 \%$ ). El precio objetivo para finales de 2019 no es fortuito, sino que es indicador de la lógica de intervención del Estado en la economía: el mismo era el precio equivalente al precio marginal del mercado a mediados de 2016, que se derivaba del precio de paridad de importación del gas natural licuado de ese año.

Por último, para mejorar los ingresos de las distribuidoras ante la demora en la aplicación de los nuevos cuadros tarifarios, de forma análoga a lo realizado por el gobierno anterior (y que fuera criticado por la Alianza Cambiemos), a finales de 2016 se aprobó un subsidio directo a las empresas en forma de asistencia económica transitoria por un total de 3450000 pesos.

De esta manera, el incremento del precio del gas en el punto de ingreso al sistema de transporte implicó una relación indirectamente proporcional entre reducción de subsidios e incremento tarifario para los usuarios finales, lo que generó una virtual transferencia de recursos de los consumidores a las empresas, al tiempo que habilitó una reducción del déficit fiscal primario para el Estado. Entre 2016 y 2017 aumentó 78.3 \% el precio del gas en boca de pozo, mientras que los valores de las tarifas residenciales lo hicieron en un promedio de 136.3 por ciento. Pero el incremento promedio de las tarifas implica invisibilizar otro de los efectos directos de la política implementada por la Alianza Cambiemos. Mientras que el modelo regulatorio de las tres gestiones kirchneristas, aun con el incremento de 2014 procuraba privilegiar a los usuarios que menos consumieran, el nuevo modelo de eficiencia marginalista invierte la lógica e impacta más -de forma 
GRÁFICA 5. TARIFAS PARA USUARIOS DE CONSUMOS BAJO, MEDIO Y ALTO, DEL PRECIO DEL GAS NATURAL EN BOCA DE POZO Y DEL SALARIO. ARGENTINA, 2013-2017. EN DÓLARES POR MILLONES DE BTU Y DÓLARES CORRIENTES

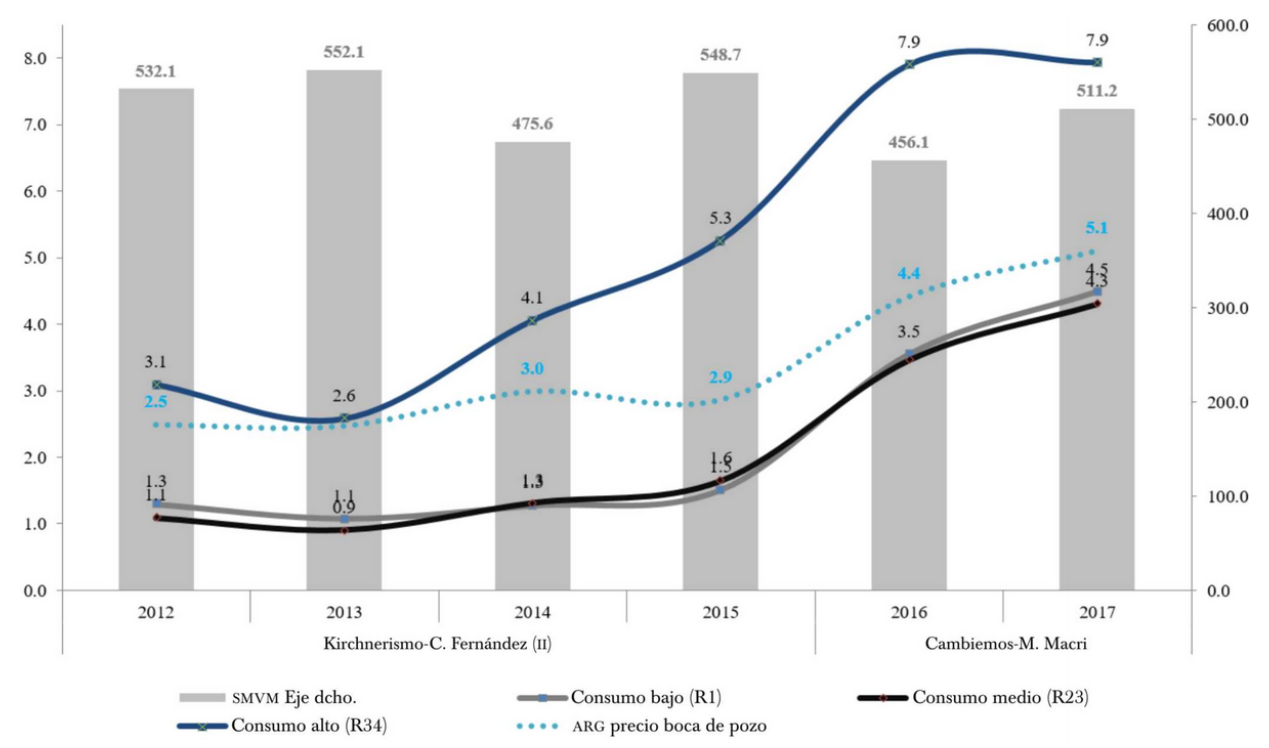

Fuentes: elaboración propia con base en Ente Nacional Regulador del Gas. Recuperado de https://www.enargas.gob.ar; Ministerio de Energía y Minería. Recuperado de https://www.argentina.gob.ar/energia y Ministerio de Trabajo, Empleo y Seguridad Social. Recuperado de https://www.argentina.gob.ar/trabajo años 2012-2017.

porcentual- a los usuarios que menos consumen: mientras las tarifas para los usuarios de mediano y bajo consumo se incrementaron $161.4 \%$ y $196.7 \%$, respectivamente, las de los de alto consumo sólo incrementaron 50.7 \% (véase gráfica 5). La regresividad distributiva del sistema se consolida en la estructura tarifaria resultante: si en 2015 el valor en dólares del millón de BTu de los usuarios de alto consumo era $248 \%$, superior a la de un usuario de bajo consumo, en 2017 la diferencia pasó a ser de 77 por ciento. En la transición de un modelo a otro, la lógica marginalista en la determinación de los precios y las tarifas hizo recaer el mayor peso de los incrementos sobre los usuarios de menor consumo.

En términos distributivos la estructura tarifaria regresiva implementada por la Alianza Cambiemos se completa con la evolución del salario mínimo que, lejos de acompañar los incrementos tarifarios, en 2017 retrocedió 6.8 \% en dólares respecto a 2015. Si se compara el ejercicio realizado en el apartado anterior en este nuevo periodo, se constata la orientación de la intervención del Estado en el sector energético: con un consumo de 180 metros cúbicos de gas natural, un usuario de consumo medio debió destinar 29 dólares en 2017 (un valor 164\% mayor que en diciembre de 2015), teniendo que utilizar 5.6\% del salario cuando en 2015 hubiese pagado once dólares y destinado $2 \%$ del mismo.

En efecto, el modelo regulatorio que emergió en diciembre de 2015 buscó romper la inercia del congelamiento tarifario de los años anteriores, incluso incrementando de manera significativa la tarifa por encima del incremento desplegado en 2014, para volver a un modelo de costo marginal 
cuya regulación tarifaria exprese la eficiencia económica del sistema, generando un mecanismo de transferencia de ingresos a lo largo de la cadena del sector, ahora desde los usuarios finales a las empresas distribuidoras.

\section{MODELOS REGULATORIOS COMPARADOS Y EFEGTOS SOBRE LAS EMPRESAS}

$\mathrm{Al}$ incorporar un análisis de largo plazo, se permite mostrar un conjunto de marchas y contramarchas en los mecanismos de regulación del servicio público de gas natural que se corresponden, vis a vis, a las distintas concepciones respecto a cuál debe ser la orientación de la intervención del Estado (véase cuadro 1 ).

Sobre los efectos de los distintos modelos regulatorios sobre las empresas, se tomó información de las revistas Mercado y Prensa Económica, publicaciones especializadas del campo empresarial de Argentina, y que desde comienzos de la década de 1970 publican anualmente el ranking de las empresas de mayor facturación. Bajo el modelo de regulación por monopolio estatal, Gas del Estado siempre estuvo dentro de las diez empresas de mayor facturación del país, incluso hasta su privatización en 1992 (véase gráfica 6). En promedio, durante la industrialización por sustitución de importaciones estuvo entre las nueve empresas, y durante la última dictadura cívico-militar, aun con la privatización periférica de empresas energéticas (Castellani y Serrani, 2010), en promedio estuvo entre las cuatro empresas de más ventas y, finalmente, desde el retorno a la democracia hasta su privatización, la empresa estuvo entre las tres primeras.

Bajo el modelo de regulación de eficiencia marginalista, las seis distribuidoras tomadas como muestra (las de mayores ventas) se mantuvieron dentro de las 200 empresas de mayor facturación entre 1992 y 2001. La combinación entre la privatización de Gas del Estado, que en 1991 era la segunda empresa de mayor facturación del país, sumado al incremento tarifario de $58.1 \%$ entre 1991 y 1993 y la dolarización de las tarifas, permitieron rápidamente el posicionamiento estructural de las distribuidoras dentro de la cúpula empresarial argentina. Sin embargo, todas descendieron en el ranking hacia finales del periodo regido por este modelo regulatorio: Metrogas, la distribuidora más grande del país, pasó del puesto 19 en 1993 al 36 en 2001, mientras que Gas Natural Fenosa y Camuzzi Pampeana cayeron del 45 al 67 y del 57 al 85, respectivamente. Entre las de menor facturación, Camuzzi Sur cayó del puesto 97 al 125, y Litoral Gas del 93 al 143. A pesar de haber tenido dolarizadas las tarifas, el atraso cambiario, producto de la convertibilidad monetaria sostenida por más de una década, impidió que las tarifas se incrementaran en moneda local, aunque sí lo hicieron en dólares corrientes (Kozulj, 2000, pp. 54-56).

En 2002, en el modelo de equidad distributiva se observa una marcada caída en las posiciones de todas las empresas, por la combinación de distintos efectos: devaluación de 237 \% y crisis económica con caída de $11 \%$ del PIB, pesificación tarifaria con caída en dólares de $58.2 \%$, que hizo retroceder $3.6 \%$ el consumo, identificándose 42000 usuarios menos conectados en relación con el año anterior. En efecto, si en promedio estas cinco distribuidoras perdieron 29 puestos entre 1993 y 2001, entre este año y 2002 la pérdida promedio fue de 43 puestos (véase gráfica 6). Con el posterior congelamiento tarifario la caída dentro del ranking de las empresas se acentuó. En promedio, 368 puestos cayeron entre 2003 y 2012 (véase gráfica 6). Dicho proceso fue matizado por el crecimiento de $26 \%$ del consumo global de gas natural y la incorporación de 1800000 nuevos usuarios ${ }^{6}$ En la etapa final de este modelo, con el FocEgAs, el incremento de 2014, y la asistencia

\footnotetext{
${ }^{6}$ Véanse datos operativos en el sitio del Enargas.
} 


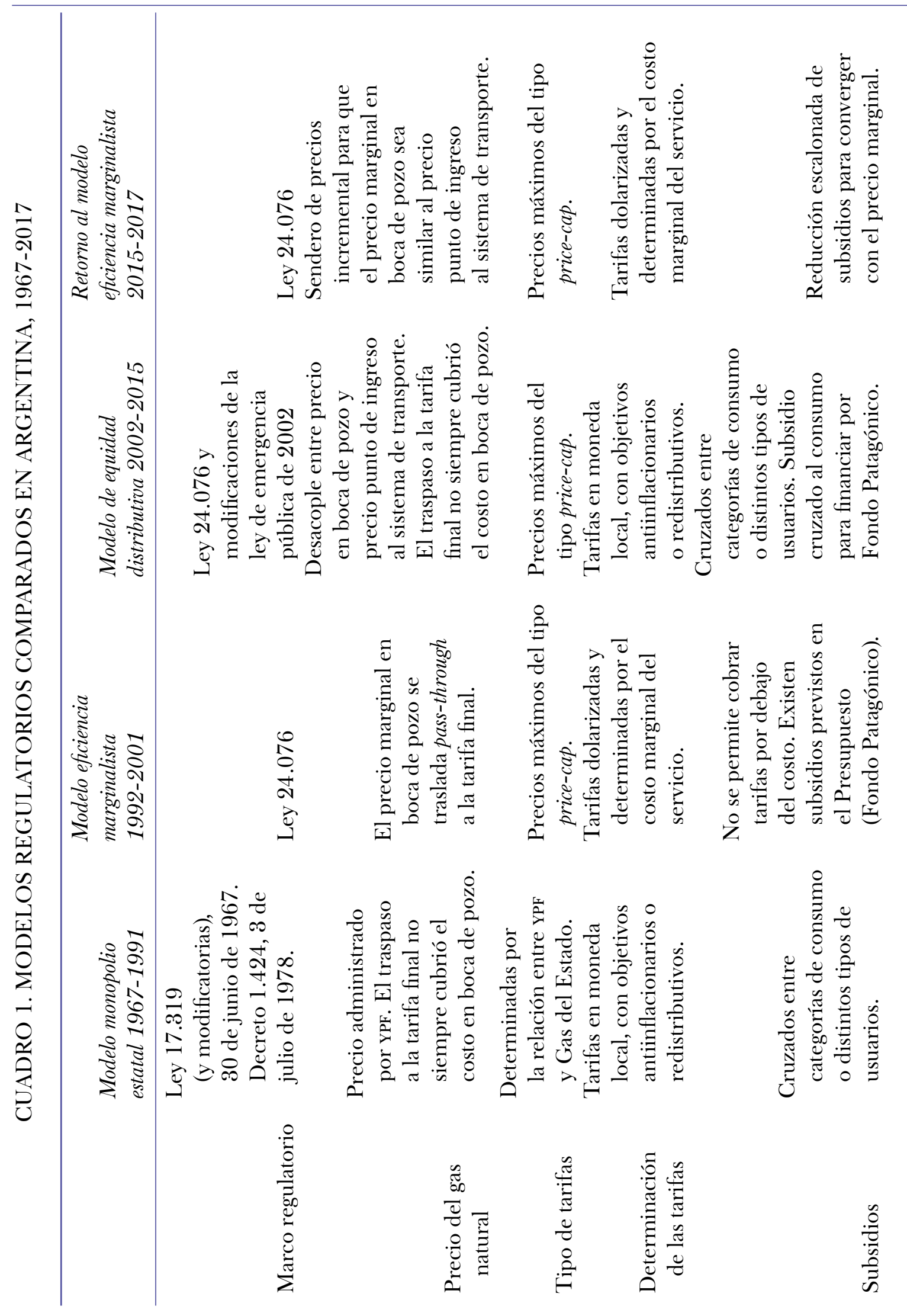



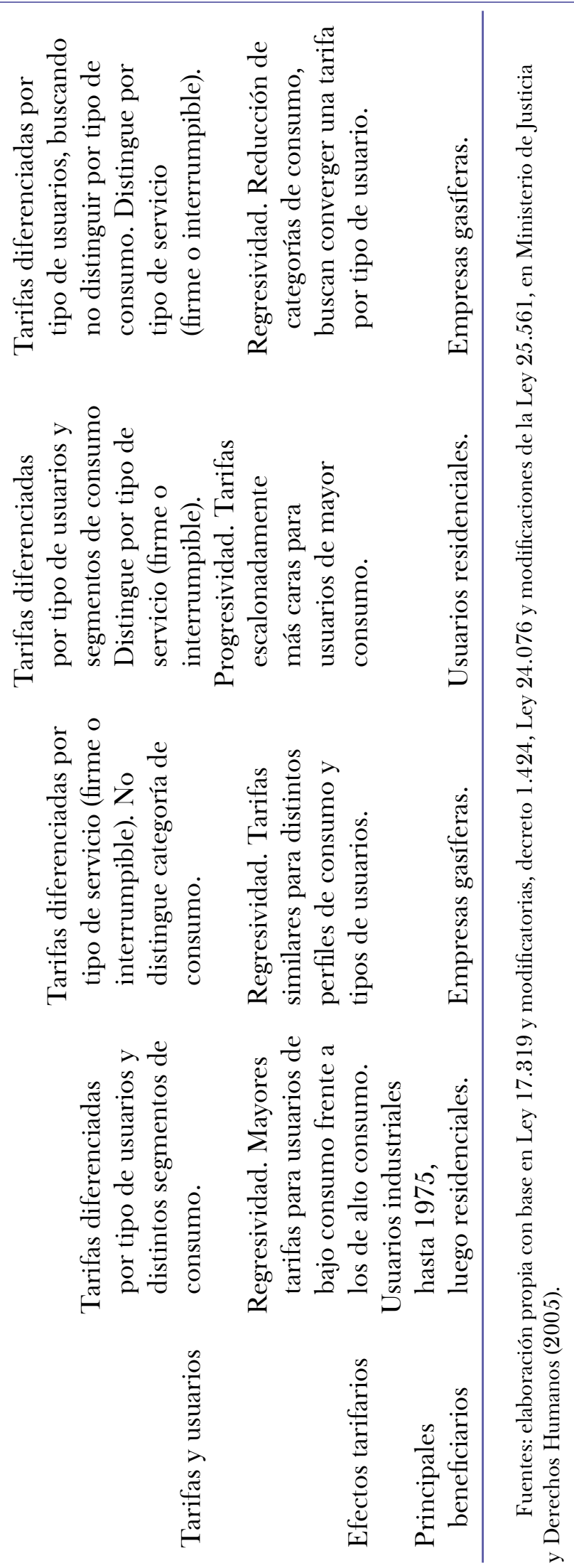


\section{GRÁFICA 6. EVOLUCIÓN DE LA PARTICIPACIÓN DE EMPRESAS DISTRIBUIDORAS DE GAS SELECGIONADAS EN EL RANKING DE LAS 1000 EMPRESAS DE MAYOR FACTURACIÓN DE ARGENTINA, 1970-2017}

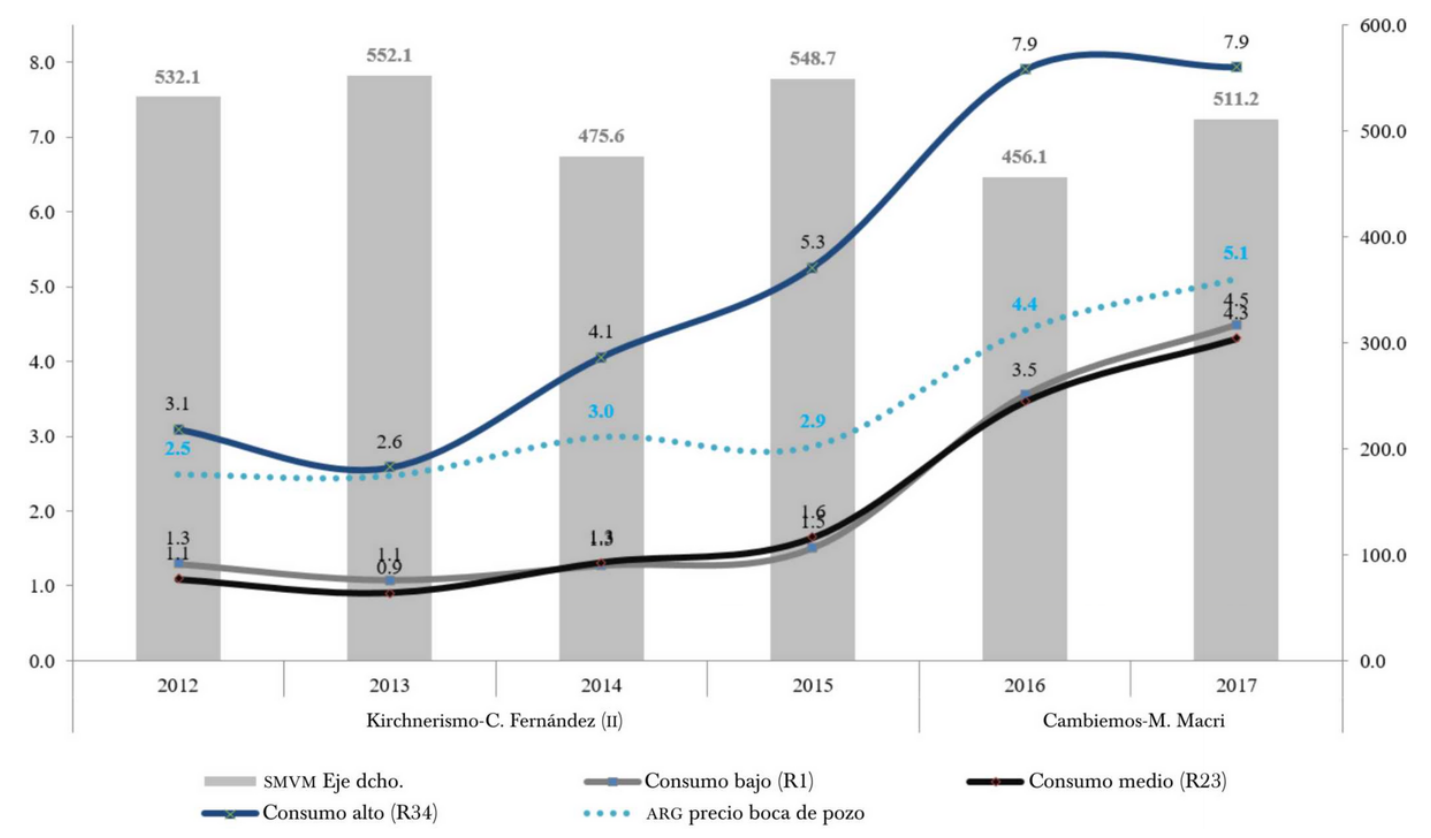

a RMEM: Retorno al modelo de eficiencia marginalista.

Fuentes: elaboración propia con base en datos de las revistas Mercado, años 1970-1992, 2003-2017. Recuperado de http://www.mercado.com.ar/23-anos-de-archivo-digital.html y Prensa Económica, años 1993-2002. Recuperado de https://www.prensaeconomica.com.ar

económica transitoria de 2015 se dio una pequeña mejora en las ventas y en el posicionamiento de las empresas en la cúpula, se recuperaron en promedio 19 posiciones entre 2015 y 2012 , entre estas, los casos de Camuzzi Pampeana (pasó de la posición 442 en 2012 a la 402 en 2015) y de Camuzzi Sur (de la 823 a la 680).

Finalmente, con el cambio de gobierno en diciembre de 2015 y el retorno al modelo de eficiencia marginalista, el incremento en las facturas finales de los usuarios se vio reflejado en una mejora sustantiva de la posición estructural de las distribuidoras entre las empresas de mayor facturación del país. En promedio recuperaron 291 posiciones en 2017 respecto a 2015, destacándose los casos de Metrogas y Gas Natural Fenosa, que vuelven a formar parte de las 200 empresas de mayor facturación del país, ocupando las posiciones 152 y 191, respectivamente, y el caso de Litoral, que avanzó 579 posiciones, de la 926 a la 347.

\section{Conclusiones}

A lo largo de este artículo se han revisado las marchas y contramarchas de los modelos regulatorios del servicio público de gas natural residencial en las últimas cinco décadas en Argentina. Los quiebres, modificaciones y readaptaciones del sistema regulatorio han estado vinculados con 
las transformaciones acontecidas en la estructura legislativa y en los mecanismos para la determinación de los precios energéticos, que han tenido diversos efectos sobre la dinámica tarifaria, el poder adquisitivo del salario y las empresas distribuidoras.

El primer modelo regulatorio abordado estuvo fundado en la administración monopólica de Gas del Estado y en su vinculación aguas arriba con ypF para el abastecimiento del gas. Más allá de las significativas diferencias respecto a los distintos partidos políticos (radicales o justicialistas) o las formas de gobierno (democráticas o dictaduras), que han administrado Gas del Estado entre 1967 y 1991, este periodo estuvo caracterizado por un marco regulatorio que tendió a generar equidad distributiva antes que buscar la eficiencia económica marginalista del sistema, en virtud de que utilizó la política tarifaria como un instrumento antiinflacionario, desacoplándolos de la dinámica de los precios internacionales (con excepción del interregno 1977-1980) y del resto de la económica local (con escaso éxito en este objetivo).

La privatización de Gas del Estado significó una profunda reestructuración del modelo regulatorio, de la morfología del sector, y de la política tarifaria respecto al modelo de regulación por monopolio de Estado. Sin embargo, desde entonces puede encontrarse un conjunto de contramarchas muy marcadas entre los modelos regulatorios que se fueron alternando.

En primer lugar, la política de dolarización tarifaria, que signó la estrategia de negocios de las empresas privatizadas de servicios públicos durante la década de 1990, se vio alterada durante todo el periodo de transición tarifaria después de 2002, y fue retomada recién en 2016, con serias dificultades para su implementación. Esto está asociado al segundo proceso, que es el quiebre del pass-through en la tarifa final, tanto por las alteraciones entre la evolución del precio en boca de pozo y el del punto de ingreso al sistema de transporte como por la interrupción de los mecanismos de revisiones periódicas para la actualización de costos y su pasaje a la tarifa final en los componentes de transporte y distribución. En tercer lugar, la política de subsidios a la tarifa final también tuvo marchas y contramarchas a pesar de la expresa determinación en el marco regulatorio del gas, que impide los subsidios cruzados entre distintos tipos de usuarios (precios de gas natural en el punto de ingreso al sistema de transporte diferentes entre residenciales e industriales, por ejemplo) o entre usuarios de distintas zonas geográficas (los usuarios patagónicos son subsidiados, en parte, por el resto de los usuarios finales residenciales a través del cargo que financia el Fondo Patagónico). Desde un modelo de administración de precios de transferencia entre dos empresas estatales a otro que quitó los subsidios en la década 1990, pasando por un periodo de ampliación de subsidios para disociar el precio de la oferta (gas en boca de pozo y gas importado) del precio de la demanda (precio punto de ingreso al sistema de transporte ), hasta la reconstrucción de un sendero de precios en el punto de ingreso al sistema de transporte con reducción escalonada de subsidios, son contramarchas que marcan las distintas formas en que cada modelo regulatorio ha manejado la determinación tarifaria. En cuarto lugar, es menester reflexionar sobre cómo cada modelo regulatorio permite interpretar diferentes concepciones respecto a cuál debe ser el papel de la energía en el entramado económico desde una perspectiva más amplia. Mientras en los modelos de regulación por eficiencia económica el factor definitivo para la determinación tarifaria fue la representación del costo marginal en la tarifa, independientemente del ritmo, evolución o trayectoria del resto de la economía, tanto en el modelo de monopolio estatal como en el de equidad distributiva el determinante para la fijación tarifaria contempló la necesidad de contar con energía barata para favorecer el crecimiento económico y no deteriorar los ingresos de los usuarios, aun cuando esta política de control de los precios de adquisición o de congelamiento 
tarifario terminó agravando las finanzas de Gas del Estado, en el primer caso, como problemas estructurales de restricción externa por la dependencia de importaciones energéticas para cubrir la brecha entre oferta y demanda, en el segundo.

Sin embargo, es necesario destacar tres continuidades que se han mantenido desde 1992 y que son el fundamento institucional actual de la configuración regulatoria de este servicio público. En primer lugar, las licencias no fueron revertidas, sino que, con las distintas revisiones que se hicieron en la década de 1990, en el periodo de transición (2002-2015) y con la última revisión tarifaria integral de 2016, todas fueron renovadas a las mismas empresas y por el plazo máximo que fija la ley. En segundo lugar, con las trasformaciones regulatorias y tarifarias derivadas de la ley de emergencia pública de 2002, y aun con el retorno al modelo de regulación marginalista de 2016, el marco regulatorio fijado por la Ley 24.076 (y sus modificatorias organizadas en lo que en este artículo se denominó marco regulatorio del gas) siguió rigiendo la presentación del servicio durante todo el periodo. Por último, si bien se transformó la lógica del pass-through en la cadena gasífera, la determinación tarifaria de distribución de gas natural siguió regida bajo el modelo de price-cap, producto de las reformas de 1992.

En efecto, no cabe duda que las persistentes marchas y contramarchas en la orientación del modelo regulatorio del servicio de gas natural en las últimas cinco décadas en Argentina ha implicado transformaciones muy profundas en su naturaleza, en sus mecanismos regulatorios, y en sus objetivos estratégicos. La falta de estabilidad regulatoria de largo plazo, el pasaje de un modelo a otro sin mediaciones ni etapas de transición lógicamente establecidas y acotadas en el tiempo, tuvieron impactos especialmente negativos en la determinación de qué precio es razonable pagar y cuáles son las características que este servicio público debería brindar para asumir que es de una calidad aceptable. Resulta necesaria una revisión sustantiva de los precios de la energía en el sector y de su normativa regulatoria, asociado a qué características y cuáles son los objetivos deseables para el largo plazo de un servicio público tan relevante en la sociedad argentina, el cual a muchas familias les resulta indispensable de acceder para subsistir en los meses más duros del invierno.

\section{LISTA DE REFERENCIAS}

Armstrong, M., Cowan, S. y Vickers, J. (1994). Regulatory reform: economic analysis and British experience. Cambridge: мIT Press.

Arroyo, M. (2015). Redes técnicas e infraestructuras urbanas. En V. Casals y L. Urteaga (eds.), Horacio Capel, geógrafo (pp. 81-107). Barcelona: Universitat de Barcelona.

Arroyo, M. y Matos, A. (2009). La modernización de dos ciudades: las redes de gas de Barcelona y Lisboa, siglos XIX y xx. Scripta Nova. Revista Electrónica de Geografía y Ciencias Sociales, 13(296, 6). Recuperado de http://www.ub.es/geocrit/sn/sn-296/sn-296-6.htm

Barrera, M. (2014). La entrega de YPF: análisis del proceso de privatización de la empresa. Buenos Aires: Atuel.

Barrera, M. A. y Serrani, E. (2018). Energía y restricción externa en la Argentina reciente. Realidad Económica, 315, 9-45.

Basualdo, E. M. (2006). Estudios de historia económica argentina: Desde mediados del siglo xx a la actualidad. Buenos Aires: Flacso/Siglo XXI Editores.

Beesley, M. E. y Littlechild, S. C. (1989). The regulation of privatized monopolies in the United Kingdom. The RAND Journal of Economics, 20(3), 454-472. DOI: 10.2307/2555582 
Bös, D. (1994). Pricing and price regulation: an economic theory for public enterprises and public utilities. Ámsterdam: Elsevier.

Bour, E. (1993). El programa argentino de desregulación y privatización. En F. A. M. de la Balze (ed.), Reforma y convergencia: ensayos sobre la transformación de la economía argentina (pp. 225272). Buenos Aires: Manantial.

Bustos, A. y Galetovic, A. (2002). Regulación por empresa eficiente: ¿quién es realmente usted? Estudios Públicos, 145-183.

Carmona, J. (1999). Galicia en el desarrollo del sector eléctrico español (1900-1982). En La industrialización y el desarrollo económico de España. Homenaje a Jordi Nadal (vol. 2, pp. 1378-1397). Barcelona: Universidad de Barcelona.

Carmona, J. y Pena, J. (1985). As origens do sector eléctrico na Galiza, 1888-1936. Agália. Revista da Associaçom Galega da Lingua, 33-48.

Castellani, A. y Serrani, E. (2010). La persistencia de los ámbitos privilegiados de acumulación en la economía argentina. El caso del mercado de hidrocarburos entre 1977 y 1999.H-Industri@, $6,2-31$.

Cayón, F. (2001). Electricidad e historia: la perspectiva de un siglo. TST: Transportes, Servicios y Telecomunicaciones, 1, 113-133.

Estache, A., Guasch, J. L. y Trujillo, L. (2003). Price caps, efficiency payoffs, and infrastructure contract renegotiation in Latin America, 3129. Recuperado de http://hdl.handle.net/10986/18130

Facultad Latinoamericana de Ciencias Sociales [Flacso] (1998). Privatizaciones en la Argentina. Marcos regulatorios tarifarios y evolución de los precios relativos durante la convertibilidad, 4, p. 111. Recuperado de http://publicacioneseconomia.flacso.org.ar/images/pdf/196.pdf11

Fernández, A. (2017). L'arrivée précoce du gaz naturel à Bordeaux (1949-1962). TST: Transportes, Servicios y Telecomunicaciones, 32, 14-26.

Fernández, A. (2018). Un yacimiento de gas natural en el origen de la formación de una región industrial del suroeste de Francia: Lacq-Mourenx (1957-2013). Revista de Historia Industrial, 27(71), 35-54.

Fernández-Paradas, M. (2005). El alumbrado público en la Andalucía del primer tercio del siglo xx: una lucha desigual entre gas y la electricidad. Historia Contemporánea, 31, 601-621.

Fernández-Paradas, M. (2018). Presentación. La industria del gas en Europa. Revista de Historia Industrial, 27(71), 11-12.

Fundación de Investigaciones Económicas Latinoamericanas [FIEL] (1998). La regulación de la competencia y de los servicios públicos: Teoría y experiencia argentina reciente. Buenos Aires: Autor.

Fundación de Investigaciones Económicas Latinoamericanas [FIEL] (1987). El fracaso del estatismo: una propuesta para la reforma del sector público argentino. Buenos Aires: Sudamericana/Planeta.

Giuntini, A. (2009). La parábola del gas in Italia dal carbone al metano dalle origini ottocentesche ad oggi. Aspetti economici, tecnologici e finanziari in chiave comparativa. TST: Transportes, Servicios y Telecomunicaciones, 16, 38-60.

Kozulj, R. (2000). Resultados de la reestructuración de la industria del gas en la Argentina. Recuperado de http://hdl.handle.net/11362/6368

Kozulj, R. y Pistonessi, H. (1989). Politicas de precios de la energía en Argentina, 1970-1989. Precios de gas natural y derivados. Bariloche: Fundación.

Laffont, J. J. y Tirole, J. (1986). Using cost observation to regulate firms. Journal of Political Economy, 94(3, parte 1), 614-641. 
Laffont, J.-J. y Tirole, J. (1993). A theory of incentives in procurement and regulation. Cambridge: MIT Press.

Littlechild, S. C. (1983). Regulation of British telecommunications' profitability report to the Secretary of State. Londres: Department of Industry.

Littlechild, S. C. (2003). The birth of RPI-X and other observations. En CRI proceedings: vol. 31. The UK model of utility regulation (pp. 31-49). Bath: The University of Batch, School of Management.

Martínez, A. y Mirás, J. (2018). Difusión y consumo de gas y electricidad para alumbrado en las urbes españolas durante la segunda transición energética (1901-1934). Revista de Historia Industrial, 27(71), 87-119.

Matos, A. C. (2018). From Belém to Marvila: the transfer of the gas plant and changes in the production process (1887-1957). Revista de Historia Industrial, Economia y Empresa, 27(71), 15-34.

Ministerio de Justicia y Derechos Humanos (2005). InfoLEG (Información Legislativa y Documental). Recuperado de http://www.infoleg.gob.ar

Moyano, F. (2012). La hulla: auge y declive en la industria del gas catalana y española. El caso de la fábrica de gas de Reus (1854-1969). TST: Transportes, Servicios y Telecomunicaciones, 22, 112-149.

Muras, R. et al. (2015). Los subsidios energéticos en Argentina. Recuperado de: http://web.iae.org.ar/wpcontent/uploads/2015/12/Informe-Final-SUBSIDIOS-ASAP-MOSCONI-VF.pdf

Posner, R. A. (1999). Natural monopoly and its regulation. Washington, D. C: Cato Institute.

Pucciarelli, A. R. (ed.) (2011). Los años de Menem: la construcción del orden neoliberal. Buenos Aires: Siglo XXI Editores.

Serrani, E. (2013). América Latina y su política petrolera frente a las últimas tendencias internacionales: perspectivas regionales a partir del análisis de Brasil y Argentina. Foro Internacional, 53(1), 182-213.

Serrani, E. (2018). Transformaciones tarifarias y efectos en la región patagónica. Camuzzi Gas del Sur y la distribución de gas natural, 1999-2017. Revista Identidades, 14(8), 45-70.

Serrani, E. y Barrera, M. A. (2018). Efectos estructurales de la política energética en la economía argentina, 1989-2014. Sociedad y Economía, 34, 122-142. DoI: 10.25100/sye.v0i34.6482

Sudrià, C. (1983). Notas sobre la implantación y el desarrollo de la industria del gas en España, 1840-1901. Revista de Historia Económica/Journal of Iberian and Latin American Economic History, 1(2), 97-118. DoI: 10.1017/S0212610900012696

Sudrià, C. (1990). La industria eléctrica y el desarrollo económico de España. En J. L. García (ed.), Electricidad y desarrollo económico: perspectiva histórica de un siglo (pp. 149-184). Oviedo: Hidroeléctrica del Cantábrico.

Waddams, C. y Zhu, M. (2013). Pricing in the UK retail energy market, 2005-2013. Working paper 13-12, East Anglia, Reino Unido. 